\title{
A WAVELET FILTER BANK WHICH MINIMIZES A NOVEL TRANSLATION INVARIANT DISCRETE UNCERTAINTY MEASURE
}

\author{
P. Tay, J.P. Havlicek, and V. DeBrunner \\ School of Electrical and Computer Engineering \\ University of Oklahoma, Norman, OK USA \\ \{ptay, joebob, vdebrunn\}@ou.edu
}

\begin{abstract}
We develop a novel measure of joint time-frequency localization applicable to equivalence classes of finite-length discrete signals, which are of increasing importance in modern signal and image processing applications. Like the wellknown Heisenberg-Weyl uncertainty principle that quantifies joint localization for continuous signals, this new measure is translation invariant and admits an intuitively satisfying interpretation in terms of the statistical variance of signal energy in time or space and in frequency. The new measure is used to design a new low-pass wavelet analysis filter with optimal joint localization. This new filter is then used to construct a localized separable 2-D discrete wavelet transform which is demonstrated on several images of general interest in practical applications.
\end{abstract}

\section{INTRODUCTION}

The Heisenberg-Weyl uncertainty principle (HUP) applied to continuous signals by Gabor quantified and conceptualized the notion of uncertainty. This principle has been the cornerstone of the uncertainty measures used in modern time-frequency analysis. In [1], Gabor defined the HUP only for functions $h: \mathbb{R}-\rightarrow \mathbb{C}$ that are both continuous ( $h$ $\left.\in C^{1}\right)$ and approach zero in the tails $\left(\lim _{t \rightarrow \pm \infty} h(t)=0\right)$. For such functions, the Heisenberg-Weyl uncertainty relation states that

$$
\Delta_{t} \Delta_{f} \geq \frac{1}{2}
$$

where the pulse width $\Delta_{t}^{2}$ is defined as

$$
\Delta_{t}^{2}=\frac{\int_{-\infty}^{\infty}(t-\langle t\rangle)^{2}|h(t)|^{2} d t}{\int_{-\infty}^{\infty}|h(t)|^{2} d t}
$$

with the mean in time $\langle t\rangle$ defined by

$$
\langle t\rangle=\frac{\int_{-\infty}^{\infty} t|h(t)|^{2} d t}{\int_{-\infty}^{\infty}|h(t)|^{2} d t} .
$$

Similarly, the band width $\Delta_{f}^{2}$ is defined as

$$
\Delta_{f}^{2}=\frac{\int_{-\infty}^{\infty}(f-\langle f\rangle)^{2}|H(f)|^{2} d f}{\int_{-\infty}^{\infty}|H(f)|^{2} d f}
$$

where the mean frequency $\langle f\rangle$ is given by

$$
\langle f\rangle=\frac{\int_{-\infty}^{\infty} f|H(f)|^{2} d f}{\int_{-\infty}^{\infty}|H(f)|^{2} d f}
$$

and where $H(f)$ is the continuous-time Fourier Transform of $h(t)$.

The HUP (1) quantifies the fact that continuous-time and continuous-frequency localization cannot be arbitrarily small simultaneously. Because of the its statistical nature, this measure is invariant to translations in time and in frequency, which agrees with our intuitive notion that simple shifting should not change the localization of a signal. Gabor also proved in [1] that the only functions which achieve equality in (1) are given by

$$
\psi(t)=e^{-\alpha^{2}\left(t-t_{0}\right)^{2}} e^{j\left(2 \pi f_{0} t+\phi\right)},
$$

where $\alpha, t_{0}, f_{0}, \phi \in \mathbb{R}$. This theory was extended into two dimensions by Daugman [2]. The functions (6) are now commonly referred to as Gabor elementary functions. Since they are optimally localized in both time and frequency, they have been used in numerous signal and image processing applications which require time-frequency analysis. This popularity extends even into the discrete domain where Gaussian shaped sequences have been created by sampling and by various forms of approximation.

Whereas the HUP is applicable only to $C^{1}$ continuoustime functions that approach zero in the tails, we present in this paper a novel translation invariant measure of uncertainty defined on equivalence classes of finite-length discrete-time sequences. This new measure is strikingly similar to the continuous time HUP and is distinct from the discrete uncertainty measures used in [3-6], which defined frequency uncertainty in terms of the discrete-time Fourier 
transform (DTFT). Moreover, the new measure is particularly significant in view of the fact that many modern applications are concerned exclusively with finite-length discrete signals to which the HUP cannot be applied.

Throughout the paper, our interest is in formulating a real-valued low-pass finite impulse response (FIR) filter function with optimal joint localization and using it to construct a separable 2-D discrete wavelet transform; we therefore restrict our attention to unit $\ell^{2}$-norm FIR sequences $h:[0, N-1]-\rightarrow \mathbb{R}$ for which $N$ is even and

$$
\sum_{n=0}^{N-1} h^{2}[n]=1=\frac{1}{N} \sum_{k=0}^{N-1}|H[k]|^{2},
$$

where $H[k]$ is the discrete Fourier transform (DFT) of $h[n]$.

\section{QUANTIFYING UNCERTAINTY AND LOCALIZATION}

We consider $h^{2}[n]$ to be a probability density function (pdf) describing the distribution of signal energy in discrete time and $\frac{1}{N}|H[k]|^{2}$ to be a pdf describing the distribution of signal energy in discrete frequency. Interpreting $n$ and $k$ as random variables in $[0, N-1]$, we define the time variance of $h$ as

$$
\sigma_{n, h}^{2}=\sum_{n=0}^{N-1}(n-\mu)^{2} h^{2}[n],
$$

where $\mu$ is the mean time given by $\mu=\sum_{n=0}^{N-1} n h^{2}[n]$. Since $h$ is assumed real, we compute the band width (variance in frequency) of $h$ according to

$$
\sigma_{\omega, h}^{2}=\frac{2}{N} \sum_{k=0}^{\frac{N}{2}} k^{2}|H[k]|^{2} .
$$

Since we are using these variances to design a realvalued scaling function, a well-know property of compactly supported wavelet scaling functions [7] is the condition $\sum_{n=0}^{N-1} h[n]=\sqrt{2}$. For signals of even lengths $N>2$, this condition eliminates the Kronecker delta and its dual the constant sequence from consideration. In addition, the $\frac{N}{2}$ term of the DFT of $h$ is equal to zero, i.e $H\left[\frac{N}{2}\right]=0$.

To quantify the joint localization of a length- $N$ sequence in discrete time and discrete frequency, we use the product of the variances (8) and (9) to define a new discretediscrete uncertainty measure given by

$$
\gamma_{N, h}^{2}=\sigma_{n, h}^{2} \sigma_{\omega, h}^{2}
$$

\section{EQUIVALENCE CLASSES OF FINITE SEQUENCES}

Our intuitive expectation is that simple shifting in time should not alter how concentrated or localized a signal is in time and in frequency. Therefore we desire a discrete uncertainty measure that, like the HUP, is translation invariant. However, the uncertainty measure $\gamma_{N, h}^{2}$ in (10) is clearly not shift invariant. In precise terms, this means that the statement

$$
h[n]=g[(n-m) \bmod N]
$$

does not necessarily imply that, for all $m \in \mathbb{Z}$,

$$
\gamma_{N, g}^{2}=\gamma_{N, h}^{2} .
$$

Here, it is understood that shifting for a finite-length sequence is defined to be circular shifting (also known as rotation).

Our approach for making the uncertainty measure (10) shift invariant is to define it not on sequences, but rather on equivalence classes of sequences. Let $h[n]$ and $g[n]$ be two real-valued sequences of length $N$. Define a relation between the two sequence as $h \sim g$ if $\exists m \in \mathbb{Z}$ such that $h[n]=g[(n-m) \bmod N]$. Simply stated, $g$ is obtained by rotating $h$ to the right by $m \bmod N$. It is obvious that this relation is reflexive, symmetric, and transitive and therefore defines an equivalence relation on the set $\mathbb{S}=\{h \mid h$ is a length $N$ sequence $\}$. For a sequence $h \in \mathbb{S}$ we define the equivalence class $[h]$ according to $[h]=\{g \in \mathbb{S} \mid g \sim h\}$, which leads to the following elegant theorem.

Theorem 1 Let $h \in \mathbb{S}$ and $g \in[h]$. Then $|H[k]|=|G[k]|$ $\forall 0 \leq k \leq N-1$.

Proof of the theorem follows easily from the "time shift property" of the DFT, which is commonly available in digital signal processing (DSP) textbooks.

To obtain a shift invariant uncertainty measure for a sequence $h \in \mathbb{S}$, we define the measure on the equivalence class $[h]$ according to

$$
\gamma_{N,[h]}^{2}=\min _{g \in[h]} \gamma_{N, g}^{2}
$$

Since Theorem 1 establishes that all members of the equivalence class $[h]$ have identical frequency variance, the uncertainty of a length- $N$ sequence $h[n]$ is then defined to be that of the member $g[n] \in[h]$ that minimizes the time variance $\sigma_{n, g}^{2}$ over $[h]$. Thus, the uncertainty measure $\gamma_{N,[h]}^{2}$ in (11) applies to all members of the class $[h]$ and is clearly shift invariant.

\section{SEARCH ALGORITHM}

A numerical optimization was implemented to determine a low-pass scaling function $h[n]$ minimizing the measure $\gamma_{N,[h]}^{2}$ in (11) for $N$ even. For the cases $N=2$ and $N=4$, the Haar scaling function is the only admissible choice for 
$h[n]$. Note that this is the only known low-pass FIR analysis filter which possesses linear phase, exact reconstruction, and orthogonality. Optimizing the phase of $H[k]$ over a wide variety of fixed magnitude responses, we observed that the minimum uncertainty filter with respect to the measure (11) always had a linear phase given by

$$
\varphi[k]= \begin{cases}\frac{\pi}{N} k & \text { for } k=0,1, \ldots, \frac{N}{2}, \\ -\frac{\pi}{N} k & \text { for } k=\frac{N}{2}+1, \ldots, N-1,\end{cases}
$$

where $H[k]=|H[k]| e^{-j \varphi[k]}$. Note that this coincides with the spectral phase of the Haar scaling function. Also, linear phase assures that a wavelet quadrature mirror filter bank can be cascaded to achieve different resolutions without the need for phase compensation.

For each even $N>4$, the search was initialized by zero padding the optimal solution from the length $N-2$ case. In the interest of computational tractability, the form (12) was assumed for the phase so that

$$
H[k]=M[k] e^{-j \varphi[k]} .
$$

The magnitude response of (13) satisfies the following conditions:

$$
\begin{aligned}
& \text { 1. } M[0]=\sqrt{2}, \\
& \text { 2. } M\left[\frac{N}{2}\right]=0, \\
& \text { 3. } M[k]=M[N-k] \text { for } k=1,2, \ldots, \frac{N}{2}-1, \\
& \text { 4. } M\left[\frac{N}{2}-1\right]=\sqrt{\frac{N-2}{2}-\sum_{k=1}^{\frac{N-4}{2}} M^{2}[k]} .
\end{aligned}
$$

The numerical procedure used a variational approach to determine an optimal set of frequency samples $H[k], k=$ $1,2, \ldots, \frac{N-4}{2}$, minimizing the uncertainty measure (11).

The optimal length $N=6$ filter may be interpreted as a generalization of the Haar function that relaxes the conditions of perfect reconstruction and orthogonality to achieve improved joint localization. The low-pass analysis filter coefficients are given by

$$
\begin{array}{lll}
h[0]= & -0.0308556756313 & =h[5] \\
h[1]= & 0.03226648753395 & =h[4] \\
h[2]= & 0.70569596928391 & =h[3] .
\end{array}
$$

For this filter, the joint uncertainty is

$$
\gamma_{6,[h]}^{2}=0.27598499451912 .
$$

For $N=8$ the search algorithm produced the following low-pass analysis filter:

$$
\begin{aligned}
& h[0]=-0.0131588203123=h[7] \\
& h[1]=-0.0284383229781=h[6] \\
& h[2]=0.04364111877368=h[5] \\
& h[3]=0.70506280570341=h[4]
\end{aligned}
$$

For this length $N=8$ filter, the joint uncertainty is

$$
\gamma_{8,[h]}^{2}=0.48428024692605 .
$$

The results of the search algorithm are summarized in Table 1, which, for each even filter length $N$, gives the uncertainty of the optimal filter, the uncertainty of the zero padded optimal length $N-2$ filter (used to initialize the numerical optimization), and the uncertainties of the corresponding length Haar and Daubechies low-pass analysis filters. As can be seen from the table, the optimal filters designed by the procedure described in this section exhibit significantly better joint localization than the corresponding length Haar and Daubechies filters for lengths $4 \leq N \leq 8$.

\section{EXPERIMENTAL RESULTS}

A jointly localized three-scale separable 2-D wavelet filterbank was created using the optimal length-8 low-pass analysis filter obtained in Section 4. To create the quadrature mirror filters, the high-pass analysis filter impulse response was set equal to the low-pass analysis filter time reversed and modulated by $e^{j \pi n}=-1^{n}$. The high-pass and lowpass synthesis filters were constructed by time reversing the corresponding analysis filters. Construction of the threescale 2-D filterbank followed the method described by Mallat in [8].

Original $M \times M$ images $\mathbf{I}$ were decomposed and reconstructed using this jointly localized 2-D wavelet transform. The reconstructed images $\mathbf{J}$ were subjectively compared with the originals for visual artifacts such as ringing, blurring, smearing, and contrast degradation. In addition, the mean squared error ( $M S E$ ) was used to quantify the filterbank performance. The $M S E$ was computed according to

$$
M S E=\frac{1}{M^{2}} \sum_{m, n=0}^{M-1}[I(m, n)-J(m, n)]^{2} .
$$

Fig. 1(a) shows the well-known original Lena image. The reconstructed Lena image $\mathbf{J}$ is given in Fig. 1(b). When evaluated at full resolution, no evident degradation in the reconstructed image is visible. The $M S E$ of the reconstructed image when compared with the original is 9.94 (out of 256 possible gray levels). Fig. 1(c) gives the histogram of the original image in Fig. 1(a), while the histogram of the reconstructed image is given in Fig. 1(d). While the histogram of the reconstructed image is not identical to the that of the original Lena image, it is evident that the shape of the histogram has been preserved.

Barbara, another well-known original image, is shown in Fig. 2(a). The reconstructed image $\mathbf{J}$ appears in Fig. 2(b). The reconstruction does not show any apparent visible 


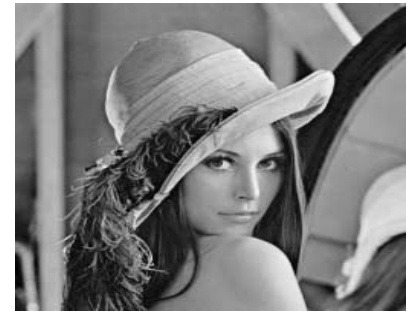

(a)

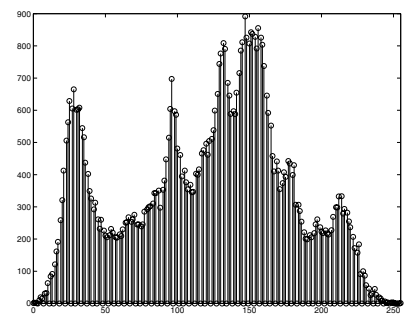

(c)

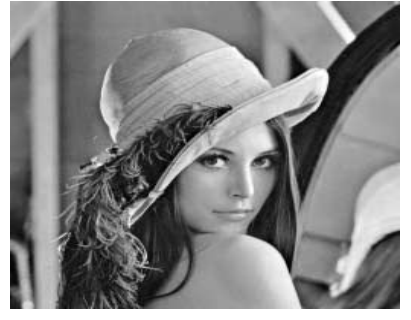

(b)

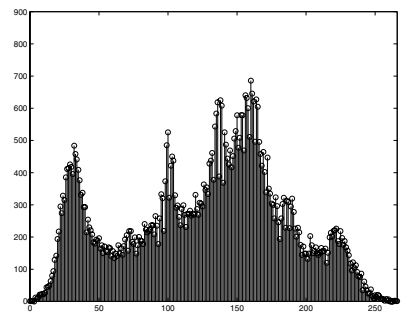

(d)

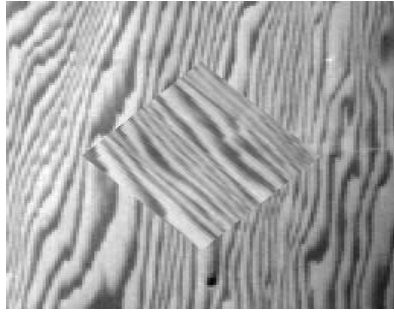

(a)

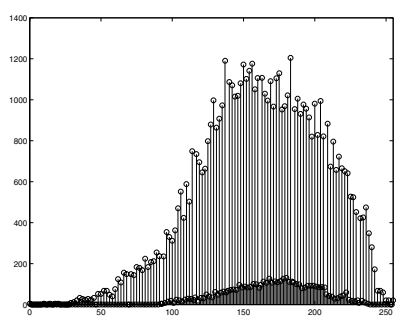

(c)

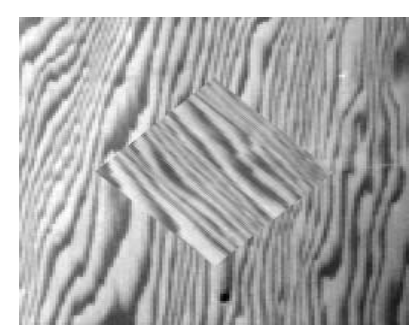

(b)

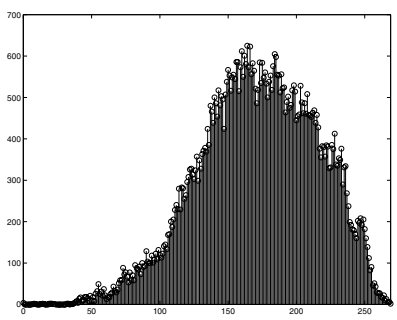

(d)

Fig. 1. Lena image. (a) Original image I. (b) Reconstructed image $\mathbf{J}$. (c) Histogram of original image I. (d) Histogram of reconstructed image $\mathbf{J}$.

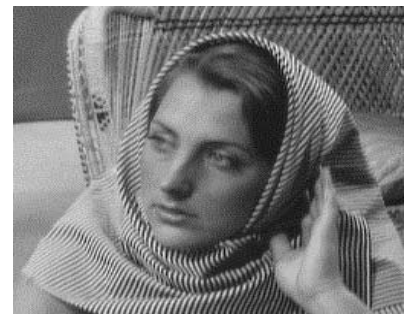

(a)

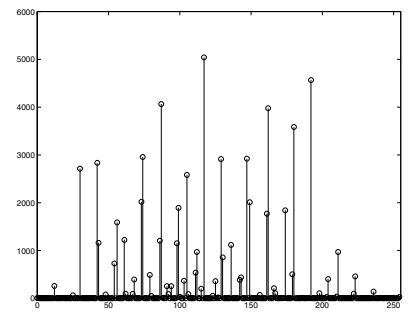

(c)

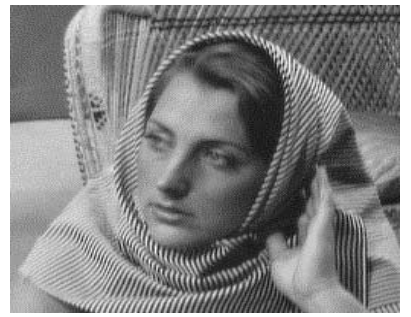

(b)

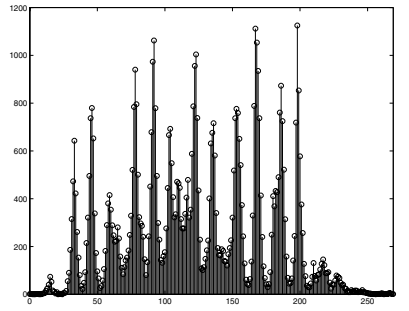

(d)

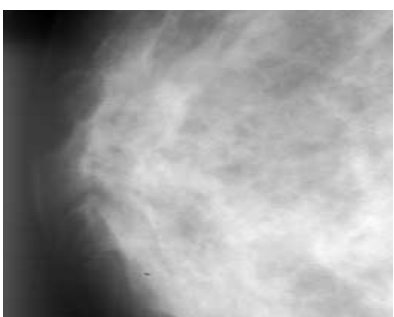

(a)

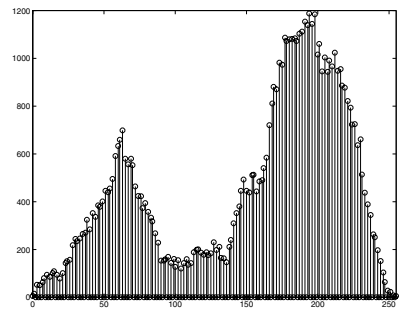

(c)

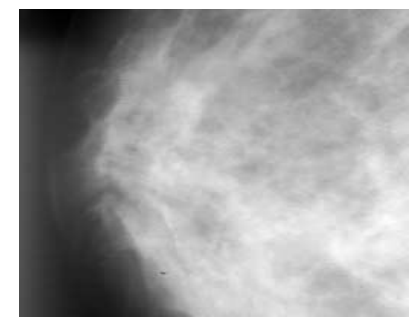

(b)

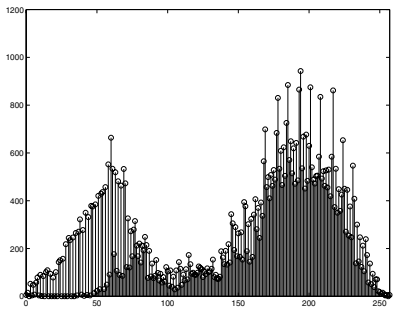

(d)

Fig. 2. Barbara image. (a) Original image I. (b) Reconstructed image J. (c) Histogram of original image I. (d) Histogram of reconstructed image $\mathbf{J}$.

Fig. 4. Mammogram image. (a) Original image I. (b) Reconstructed image J. (c) Histogram of original image I. (d) Histogram of reconstructed image $\mathbf{J}$. 


\begin{tabular}{||c|c|c|c|c||}
\cline { 2 - 5 } \multicolumn{1}{c|}{} & \multicolumn{4}{c||}{ Uncertainty } \\
\hline \hline $\begin{array}{c}\text { Filter } \\
\text { Length } \\
N\end{array}$ & $\begin{array}{c}\text { Optimal } \\
\text { Lenght } N \\
\text { Filter }\end{array}$ & $\begin{array}{c}\text { Optimal Length } \\
N-2 \text { Filter, } \\
\text { Padded }\end{array}$ & $\begin{array}{c}\text { Length } N \\
\text { Haar } \\
\text { Filter }\end{array}$ & $\begin{array}{c}\text { Dau- } \\
\text { bech } \\
\text { ies }\end{array}$ \\
\hline \hline 2 & 0.00000 & 0.0000 & 0.0000 & 0.00000 \\
\hline 4 & 0.12500 & 0.1250 & 0.1250 & 0.15180 \\
\hline 6 & 0.27590 & 0.2917 & 0.2917 & 0.39580 \\
\hline 8 & 0.48430 & 0.4921 & 0.5214 & 0.99230 \\
\hline \hline
\end{tabular}

Table 1. Uncertainty measure for even filter lengths $2 \leq$ $N \leq 8$. The second column gives the uncertainty $\gamma_{N,[h]}^{2}$ for the optimal filter designed by the technique described in Section 4. The third column gives the uncertainty of the initial filter for each length $N$, which was obtained by zero padding the optimal filter found for length $N-2$. For comparison, the last two columns give uncertainty measures for the Haar and Daubechies low-pass analysis filters of corresponding length.

degradation at full resolution. The $M S E$ for this example is 6.0739. Although the histogram of the original image shown in Fig. 2(c) differs noticeably from the histogram of the reconstruction in Fig. 2(d), the peaks of the two histograms are in good correspondence.

The original WoodWood image shown in Fig. 3(a) was obtained by rotating the central portion of a homogeneous wood grain textured image. The reconstructed image $\mathbf{J}$ is shown in Fig. 3(b) and exhibits no visible artifacts; the $M S E$ is 7.3791. Histograms of the original image $\mathbf{I}$ and reconstructed image $\mathbf{J}$ are given in Fig. 3(c) and (d), respectively, and appear quite similar.

Finally, the original image Mammogram is shown in Fig. 4(a). The reconstruction $\mathbf{J}$ is given in Fig. 4(b), where the $M S E$ was 0.5172 . Once again, no visible artifacts were detected upon visual inspection of the full resolution reconstructed image. While the values of the original and reconstructed histograms shown in 4(c) and (d), resp., vary substantially, the bimodal nature of the gray level distribution is preserved.

\section{CONCLUSION}

We introduced a novel measure of joint uncertainty applicable to real-valued FIR filters of even finite lengths. This new uncertainty measure is analogous to the continuous-time Heisenberg-Weyl uncertainty measure in that it admits an intuitive interpretation of time and frequency localization as simple statistical variances and is also invariant under time translation. The translation invariance property was obtained by defining the measure not on discrete-time signals themselves, but rather on equivalence classes of discretetime signals. Variances in time and frequency were defined with respect to the natural probability distribution of signal energy in each domain inherited from the $\ell^{2}$-norm of an FIR filter and its DFT as opposed to the DTFT.

A numerical optimization algorithm was employed to design low-pass analysis filters with optimal joint localization for lengths $N=6$ and $N=8$. These new filters exhibited significantly improved joint uncertainty as compared with the Haar and Daubechies filters of corresponding lengths. The optimal length $N=8$ filter was used to construct a jointly localized three-scale 2-D separable discrete wavelet transform that was demonstrated on four images of general interest in practical applications. Visually, the reconstructed images were free of artifacts and virtually indistinguishable from the originals in each case. While this new wavelet transform sacrificed orthogonality and perfect reconstruction to obtain optimal joint localization, we obtained an $M S E$ of less than $4 \%$ in each case. The main features of the image histograms were also preserved.

\section{REFERENCES}

[1] D. Gabor, "Theory of communication," J. Inst. Elect. Eng. London, vol. 93, no. III, pp. 429-457, 1946.

[2] J. G. Daugman, "Uncertainty relation for resolution in space, spatial frequency, and orientation optimized by two-dimensional visual cortical filters," J. Opt. Soc. Am. A, vol. 2, no. 7, pp. 1160-1169, July 1985.

[3] L. Calvez and P. Vilbé, "On the uncertainty principle in discrete signals," IEEE Trans. Circuits, Syst.-II: Analog, Digital Sig. Proc., vol. 39, no. 6, pp. 394-395, June 1992.

[4] R. Ishii and K. Furukawa, "The uncertainty principle in discrete signals," IEEE Trans. Circ. Syst., vol. CAS-33, no. 10, pp. 1032-1034, Oct. 1986.

[5] M. Doroslovački, "Discrete-time signals and uncertainty relations involving ordinary second moments in time and frequency," in Proc. IEEE Int'l. Symp. Signal Proc., Philadelphia, PA, Oct. 1994, pp. 186-189.

[6] P. Stoica and R. Moses, Introduction to spectral analysis, Prentice Hall, New Jersey, 1997.

[7] I. Daubechies, "Orthonormal bases of compactly supported wavelets," Commun. Pure Appl. Math., vol. 51, pp. 909-996, 1988.

[8] S. G. Mallat, "A theory for multiresolution signal decomposition: the wavelet representation," IEEE Trans. Pattern Anal. Machine Intell., vol. 11, no. 7, pp. 674693, July 1989. 